
A. ANDREONI ${ }^{1, \infty}$
E. PUDDU ${ }^{1}$
M. BONDANI ${ }^{2}$

\title{
Parallel spatial intensity correlations to decode random frequency-downconverted images
}

\author{
${ }^{1}$ Dipartimento di Fisica e Matematica, Universita' degli Studi dell'Insubria and Consiglio Nazionale delle \\ Ricerche, C.N.R., Istituto Nazionale per la Fisica della Materia, I.N.F.M., Via Valleggio 11, \\ 22100 Como, Italy \\ ${ }^{2}$ National Laboratory for Ultrafast and Ultraintense Optical Science (ULTRAS)-C.N.R.-I.N.F.M., \\ Via Valleggio 11, 22100 Como, Italy
}

\section{Received: 2 January 2006/Revised version: 15 April 2006 Published online: 14 July 2006 • ( ) Springer-Verlag 2006}

ABSTRACT We record frequency-downconverted images that are chaotic, as they are obtained in a $\chi^{(2)}$ crystal from the interaction of two pulsed pseudo-thermal fields of which the one at the higher frequency encountered the imaged object. Spatial correlations of the intensity fluctuations in these chaotic images with the intensity of a single spatial Fourier component of the low-frequency input field, allow image retrieval if the number of records on which the ensemble-averages are calculated is suitably large. When it is too small to achieve a satisfactory result, we show that computing the correlations in parallel with different components of the low-frequency input field, shifting the correlation maps according to a rule suggested by $3 \mathrm{D}$ phase-matching, and averaging them, leads to the recovery of the downconverted image. The method can be used for secure and fast image transmission.

PACS 42.65.Lm; 42.30.Va; 42.50.Ar

1

\section{Introduction}

Thermal light sources are receiving renewed attention in the context of the lively debate about the possibility of reproducing, with classical correlations, results obtained with quantum correlations. Bennink et al. [1] first opened this debate with their experiment of ghost imaging by means of classically correlated photon pairs obtained from a $\mathrm{He}-\mathrm{Ne}$ laser beam whose coherence was destroyed by a chopper plus moving mirror. In this debate, the successful two-photon correlated imaging experiment that was performed by Zhang et al. [2] using a true thermal light source is an important step to ascertain to which extent naturally incoherent light can emulate entangled light in ghost imaging experiments [3]. In all cases the computation of suitable correlation functions allows image retrieval. In the more general context in which such computation techniques are applied for extracting image information from chaotic intensity maps, we have recently proposed an experiment utilizing seeded downconverted correlated beams [4]. In the experiment described in [4] we mimic spontaneous downconversion as the phase of the lowfrequency input beam is made random by the insertion of

Fax: +39-031-2386119, E-mail: Alessandra.Andreoni@uninsubria.it a moving light-diffusing plate and adopt a detection protocol based on spatial correlations of the intensity fluctuations. The protocol allows us to recover the image encoded on the high-frequency beam (field $\boldsymbol{E}_{3}$ ) from the distributed image information carried by signal and idler beams that are randomized in space at each shot and made locally random from shot to shot [5]. Image recovery was obtained by correlating the spatial intensity distribution of the frequency-downconverted field (generated field $\boldsymbol{E}_{2}$ ), on the plane in which it should form the image, with the intensity of a particular Fourier component of the low-frequency input field, $\boldsymbol{E}_{1}[4,6]$.

In the present experiment we insert a diffuser not only on the $\boldsymbol{E}_{1}$ beam but also in the high-frequency beam (field $\boldsymbol{E}_{3}$ ). By using the information on the direction of $\boldsymbol{k}_{1}$, we can reconstruct the image of the object mask inserted in the path of $\boldsymbol{E}_{3}$. As (due to the phase-matching condition) the position of the reconstructed image is determined by the direction of the selected $\boldsymbol{k}_{1}$, we can substantially refine the image-recovery protocol. The new protocol includes an image enhancement procedure that fully utilizes the information on the image that is stored in the chaotic records of the spatial distribution of the frequency-downconverted field, $\boldsymbol{E}_{2}$. We demonstrate that this protocol provides a further key that allows decoding of the image even when spatial intensity correlations with any of the Fourier components of the randomized input field fail, because the number of chaotic-image records is too small. With this protocol we do not intend to improve the quality of the image by optimizing the spatial intensity correlation technique, which can be done for instance, by implementing spatial averages over the test-arm detector position $[7,8]$. We make use of the constraints set by phase matching on the spatial intensity correlations with sets of selected components of the spatial spectrum of the low-frequency input-field.

2

\section{Plan of the experiment}

The experiment is performed with the same setup as in [4] and utilizes a double slit as the object modulating the high frequency beam (second-harmonics output of a $Q$-switched Nd:YAG laser, $10 \mathrm{~Hz}$ repetition rate). As sketched in Fig. 1, the slits $(\sim 80 \mu \mathrm{m}$ aperture spaced by a blank of $\sim 530 \mu \mathrm{m}$ width) are horizontal as well as the beam at $2 \omega$ and that at $\omega$, which serves as the field $\boldsymbol{E}_{1}$ that pumps 

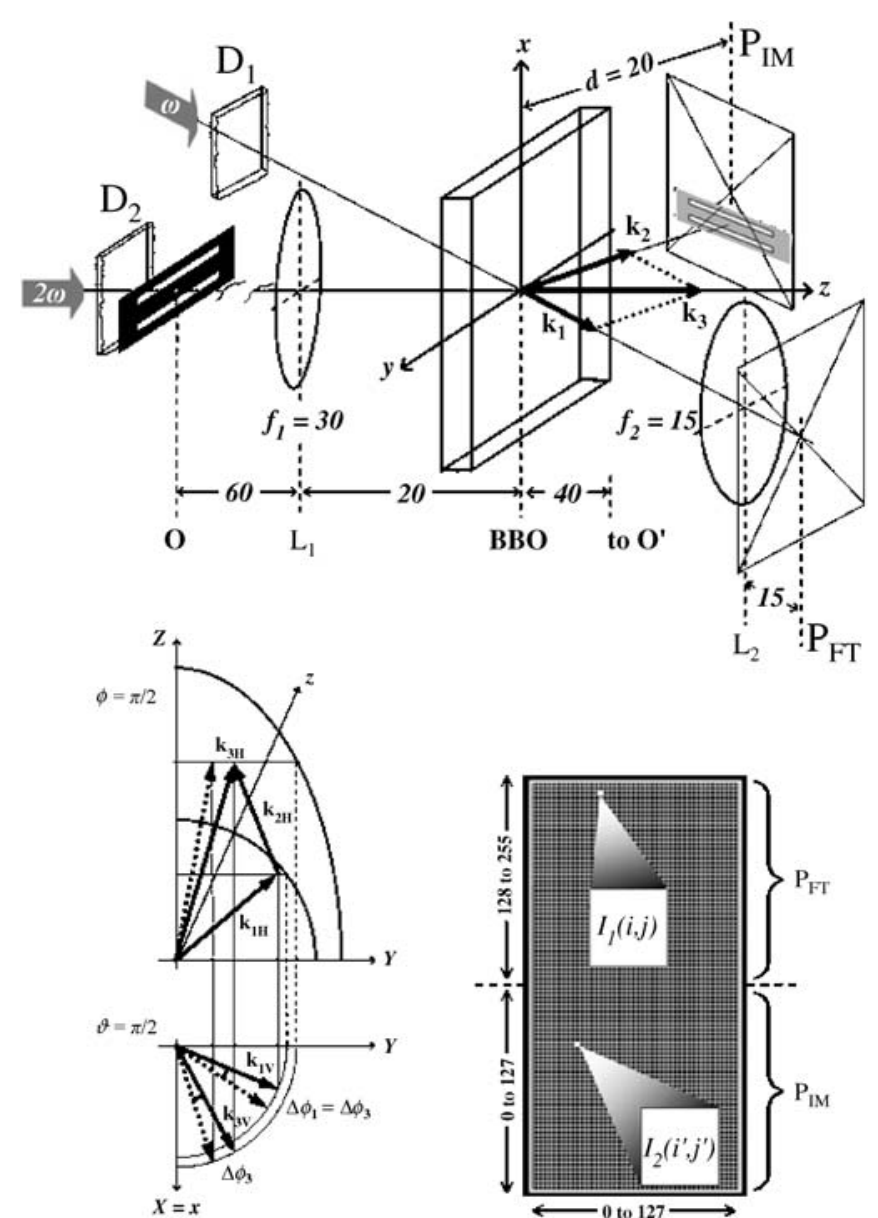

FIGURE 1 Schematic view of the image frequency-downconversion process and of the detection. All dimensions are in $\mathrm{cm}$. The BBO crystal, whose entrance face is on the $(x, y)$-plane, converts the virtual image of the back illuminated double slit, $\mathrm{O}$, that lens $\mathrm{L}_{1}$ forms in $\mathrm{O}^{\prime}$. $\mathrm{D}_{1}$ and $\mathrm{D}_{2}$ : moving ground-glass diffusing plates. $\mathrm{P}_{\mathrm{IM}}$ : plane of the downconverted real image. $\mathrm{P}_{\mathrm{FT}}$ : Fourier plane of lens $\mathrm{L}_{2}$. The $\mathrm{BBO}$ optical axis, $Z$, not shown in the main panel, lies on the $(y, z)$-plane. Lower left: projections onto the $(Y, Z)$ and $(X, Y)$-planes $(X, Y, Z$ crystal axes) of three phase-matched wave-vectors, $\boldsymbol{k}_{1}, \boldsymbol{k}_{2}, \boldsymbol{k}_{3}$ (full-line vectors) lying on a plane not containing the $Z$-axis. The $(Y, Z)$-projections $\left(\boldsymbol{k}_{1 H}, \boldsymbol{k}_{2 H}, \boldsymbol{k}_{3 H}\right)$ are horizontal, the $(X, Y)$-projections $\left(\boldsymbol{k}_{1 V}, \boldsymbol{k}_{2 V}, \boldsymbol{k}_{3 V}\right)$ are vertical. Dotted $\boldsymbol{k}_{1}$ : wave-vector phase-matched with dotted $\boldsymbol{k}_{3}$, which is rotated by $\Delta \varphi_{3}$ about $Z$ (dotted $\boldsymbol{k}_{2}$ not shown). Lower right: partition of the CCD sensor detecting the intensity distributions on $\mathrm{P}_{\mathrm{IM}}$ and that on $\mathrm{P}_{\mathrm{FT}}$ upon suitable deviation of the $\boldsymbol{k}_{1}$ beam after $\mathrm{BBO}$

the difference-frequency generation process (non-collinear type I interaction in a $\beta-\mathrm{BaBO}_{4}$ crystal, $\mathrm{BBO}$, of $10 \times 10 \times$ $4 \mathrm{~mm}$ thickness, cut at $32 \mathrm{deg}$, with the optical axis lying horizontally, $(y, z)$-plane in the laboratory reference frame). The pulse energy in either of the two beams was $\sim 1 \mathrm{~mJ}$. The moving diffusers $\mathrm{D}_{1}$ and $\mathrm{D}_{2}$ make fields $\boldsymbol{E}_{1}$ and $\boldsymbol{E}_{3}$ pseudothermal by randomizing both their propagation directions and photon-flux densities, $\left|a_{1}\right|^{2}$ and $\left|a_{3}\right|^{2}$. Under the hypothesis of negligible phase mismatch, the slit image at $2 \omega$ (virtual object image, $\mathrm{O}^{\prime}$ not shown in the figure), which forms at $40 \mathrm{~cm}$ beyond $\mathrm{BBO}$, would be downconverted by a single spatial Fourier component (wave-vector $\boldsymbol{k}_{1}$ ) of the seed field into a real image that forms at $d=20 \mathrm{~cm}$ on the plane indicated as $\mathrm{P}_{\mathrm{IM}}$ in Fig. 1 [9]. Actually the randomization caused by $\mathrm{D}_{1}$ on the $\boldsymbol{k}_{1}$ wave-vectors in direction and on the $\left|a_{1}(z=0)\right|^{2}$ values brings a chaotic intensity distribution of the generated field $\boldsymbol{E}_{2}$ on plane $\mathrm{P}_{\mathrm{IM}}$ at each laser shot. We simultaneously record the space Fourier transform of field $\boldsymbol{E}_{1}$ on plane $\mathrm{P}_{\mathrm{FT}}$ (see Fig. 1) at each of the shots used in the experiment. As detailed in the lower right panel of the figure, $\mathrm{P}_{\mathrm{FT}}$ and $\mathrm{P}_{\mathrm{IM}}$ are made to coincide on the sensor of our CCD camera so that $\mathrm{P}_{\mathrm{FT}}$ and $\mathrm{P}_{\mathrm{IM}}$ occupy the upper and lower halves of the sensor. The pixel positions are identified by couples of integer numbers $(i, j)$ on $\mathrm{P}_{\mathrm{FT}}$ and $\left(i^{\prime}, j^{\prime}\right)$ on $\mathrm{P}_{\mathrm{IM}}$. The CCD camera is a Dalsa CA-D1256T model, with $16 \mu \mathrm{m} \times 16 \mu \mathrm{m}$ pixels, 12-bit resolution, which is driven by a frame grabber synchronous with the laser shots. Each frame is a single-shot record, as the exposure time is $10 \mathrm{~ms}$ only, and thousands of recorded frames are stored.

Before presenting the experimental results we mention that according to references [4] and [6], in which we disregarded phase mismatch, when we select a single point in the $\mathrm{P}_{\mathrm{FT}}$ plane and construct the map of the spatial intensity fluctuations between the chosen $\boldsymbol{E}_{1}$ component and field $\boldsymbol{E}_{2}$ at all points in the $\mathrm{P}_{\mathrm{IM}}$ plane, we retrieve the image of the object. This image is transversally displaced with respect to the position of the downconverted image that would be obtained without diffuser $\mathrm{D}_{1}$ by using a collinear interaction $\left(\boldsymbol{k}_{1}\right.$ parallel to $\boldsymbol{k}_{3}$ before $\mathrm{D}_{2}$ ). While the latter would be recorded as an intensity distribution $\left|a_{2}\left(\bar{i}^{\prime}, \bar{j}^{\prime}\right)\right|^{2} \propto\left|a_{3}\left(\bar{i}^{\prime}, \bar{j}^{\prime}\right)\right|^{2}$, the image retrieved by calculating

$$
\begin{aligned}
& G_{i, j}\left(i^{\prime}, j^{\prime}\right)=\frac{\left\langle\Delta I_{1}(i, j) \Delta I_{2}\left(i^{\prime}, j^{\prime}\right)\right\rangle}{\sigma\left[I_{1}(i, j)\right] \sigma\left[I_{2}\left(i^{\prime}, j^{\prime}\right)\right]} \\
& \equiv \frac{\left\langle I_{1}(i, j) I_{2}\left(i^{\prime}, j^{\prime}\right)\right\rangle-\left\langle I_{1}(i, j)\right\rangle\left\langle I_{2}\left(i^{\prime}, j^{\prime}\right)\right\rangle}{\sqrt{\left[\left\langle I_{1}^{2}(i, j)\right\rangle-\left\langle I_{1}(i, j)\right\rangle^{2}\right]\left[\left\langle I_{2}^{2}\left(i^{\prime}, j^{\prime}\right)\right\rangle-\left\langle I_{2}\left(i^{\prime}, j^{\prime}\right)\right\rangle^{2}\right]}},
\end{aligned}
$$

would be proportional to $\left|a_{3}\left(i-\bar{i}^{\prime}, j-\bar{j}^{\prime}\right)\right|^{2}$ in which $\left(i-\bar{i}^{\prime}\right)$ and $\left(j-\bar{j}^{\prime}\right)$ only depend on the direction of $\boldsymbol{k}_{1}$ with respect to $\boldsymbol{k}_{3}$ (see [6] for a detailed calculation).

The lower left panel in Fig. 1 shows the key point of the present experiment. By working with a diffused highfrequency light, in the presence of a diffused low-frequency input field, we actually have difference-frequency light that reaches $\mathrm{P}_{\mathrm{IM}}$ at any position being generated in conditions of phase matching. In the crystal reference frame $(X, Y, Z)$, in which the BBO optical axis is the $Z$-axis, we consider three phase-matched wave-vectors $\boldsymbol{k}_{1}, \boldsymbol{k}_{2}$ and $\boldsymbol{k}_{3}$ lying in a plane not containing the $Z$-axis. Their projections $\boldsymbol{k}_{1 H}, \boldsymbol{k}_{2 H}$ and $\boldsymbol{k}_{3 H}$ on the $(Y, Z)$-plane (horizontal in the experiment) and those on the $(X, Y)$-plane, $\boldsymbol{k}_{1 V}, \boldsymbol{k}_{2 V}$ and $\boldsymbol{k}_{3 V}$, are shown in the lower left panel. The symmetry of the k-surfaces guarantees that a $\boldsymbol{k}_{3}$ wave-vector (dotted) rotated by any angle about the $Z$-axis remains phase matched with $\boldsymbol{k}_{1}$ and $\boldsymbol{k}_{2}$ wave-vectors rotated by the same angle ( $\Delta \varphi_{1}=\Delta \varphi_{2}=\Delta \varphi_{3}$ in the figure). Similarly, but at first order approximation only, if a $\boldsymbol{k}_{3}$ wave-vector is rotated by $d \vartheta_{3}$ about the $X$-axis, the $\boldsymbol{k}_{1}$ and $\boldsymbol{k}_{2}$ wave-vectors must rotate by $\Delta \vartheta_{1}$ and $\Delta \vartheta_{2}$ angles that are proportional to $d \vartheta_{3}$ for the fulfillment of the phase matching condition. The latter property is irrelevant for our horizontal slits, but the former one, which holds for both type I and type II interactions, allows us to extend the validity of our detection protocol based on spatial intensity correlations. By returning to the $(x, y, z)$ reference frame, which simply corresponds to rotating $(X, Y, Z)$ about $x \equiv X$ by the cut angle, and observing that 
$\mathrm{P}_{\mathrm{FT}}$ and $\mathrm{P}_{\mathrm{IM}}$ receive fields with wave-vectors $\boldsymbol{k}_{1}$ and $\boldsymbol{k}_{2}$, respectively, at small angles to normal incidence, the property of the above shows that, as $G_{i, j}\left(i^{\prime}, j^{\prime}\right) \propto\left|a_{3}\left(i-\bar{i}^{\prime}, j-\bar{j}^{\prime}\right)\right|^{2}$, for a correlation $G_{i, j+\delta}\left(i^{\prime}, j^{\prime}\right)$ we find

$G_{i, j+\delta}\left(i^{\prime}, j^{\prime}\right) \propto\left|a_{3}\left[i-\bar{i}^{\prime}, j-\left(\bar{j}^{\prime}+\delta^{\prime}\right)\right]\right|^{2}$,

in which $\delta$ and $\delta^{\prime}$ are shifts in the vertical direction (see Fig. 1). As the rotation angle corresponding to $j \rightarrow j+\delta$ must be the same as that corresponding to $\bar{j}^{\prime} \rightarrow \bar{j}^{\prime}+\delta^{\prime}$, we get:

$\frac{\delta}{f_{2}}=\frac{\delta^{\prime}}{d}$.

In our case: $\delta^{\prime} / \delta=4 / 3$.

\section{3}

\section{Results and discussion}

To verify this property we calculated 21 spatial intensity correlations according to (1) for an ensemble of 6,000 single-shot records for fixed $i=64$ and $j=231$ to 251 (step $\delta=1$ ). Note that the choice of the value of $i$ was not particularly accurate: we simply noticed that the space Fourier transform of field $\boldsymbol{E}_{1}$ recorded on plane $\mathrm{P}_{\mathrm{FT}}$ was centered at the corresponding abscissa. Note also that we extend the calculation to cover the entire sensor, so to verify that, in the upper half, we get $G_{i, j}(i, j)=1$ as expected from the normalization in (1). Actually we find $G_{i, j}\left(i^{\prime}, j^{\prime}\right)$-maps sharply peaking at pixel $(i, j)$ in which the value 1 is achieved. These 21 correlations are plotted as gray scale maps and used as frames in the movie of [10] whereas $G_{i, 231}$ is plotted in Fig. 2a, in which the image is clearly detectable as well as the auto-correlated pixel (64 231). In the recovered images the slits are separated by 35 to 40 pixels, which agrees with the $610 \mu \mathrm{m}$ center-to-center separation both of the true slits and their virtual image $(\mathrm{O}$ and $\mathrm{O}^{\prime}$, respectively, see above) [9]. For comparison, in Fig. 2b
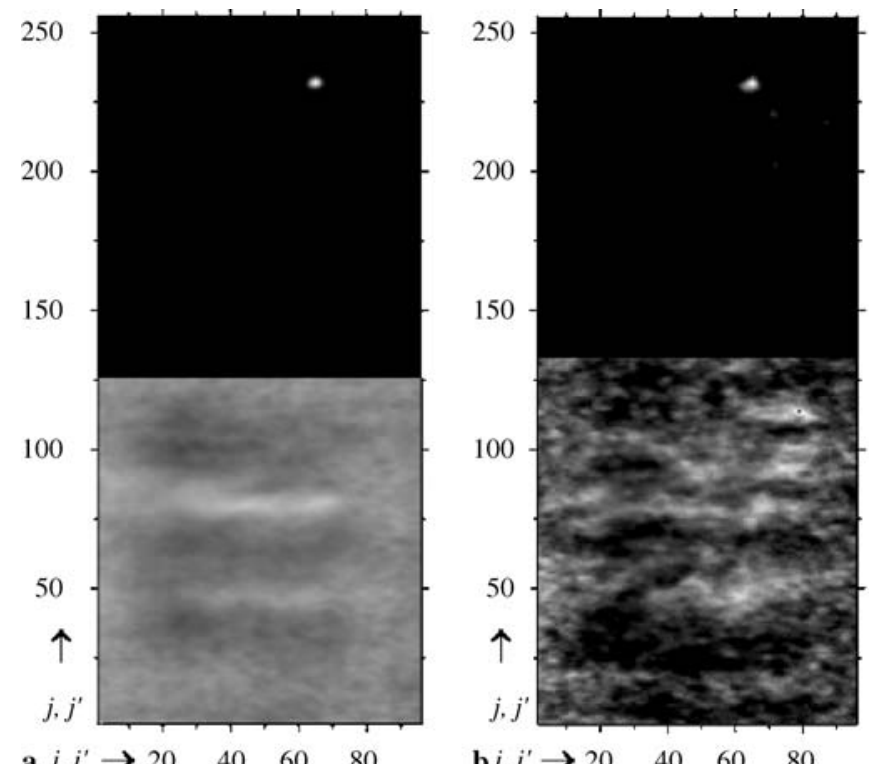

a $i, i^{\prime} \rightarrow 20 \quad 40 \quad 60 \quad 80 \quad$ b $i, i^{\prime} \rightarrow 20 \quad 40 \quad 60 \quad 80$

FIGURE 2 Maps of the spatial intensity correlation coefficients $G_{i, 231}$ (with $i=64$ ) obtained by applying (1) to an ensemble of 6000 single-shot CCD records in (a), and of 250 records in (b) we plot the correlation map $G_{i, 231}$ for a sub-set of single-shot records containing 250 instead of 6000 samples. The two plots in Fig. 2 show that limiting the size of the ensemble on which the averaging operation $\langle\ldots\rangle$ in (1) is performed returns a correlation map that is too noisy to allow image retrieval. In [10], with the help of the markers, which move with speeds in the ratio $3 / 4$, we can observe the correctness of the relation [see (2) and (3), and the Appendix] between the displacements of the auto-correlated pixel and of the recovered image. Hence, if we select the maps for $\mathrm{j}$ steps equal to 3 , we should find images shifted by four pixels in the opposite direction. We divided the 21 maps into three series, one containing $G_{i, 231}, G_{i, 234}, G_{i, 237}$, etc. and similarly for the other two, which start with $G_{i, 232}$ and $G_{i, 233}$, respectively. Upon properly shifting the lower parts of the maps by multiples of four pixels, we averaged over each series and obtained averaged spatial correlation maps, $G_{i, 231}^{\mathrm{AVE}}$, $G_{i, 232}^{\mathrm{AVE}}$ and $G_{i, 233}^{\mathrm{AVE}}$ that are displayed in panels a, b, and $\mathrm{c}$ of Fig. 3.

Further shifting $G_{i, 231}^{\mathrm{AVE}}$ and $G_{i, 233}^{\mathrm{AVE}}$ by \pm 1 and averaging with $G_{i, 232}^{\mathrm{AVE}}$ produces the map $G_{i}^{\mathrm{FINAL}}$ shown in Fig. 4a. To obtain this we have utilized all information contained in the initial 6000 frames. For the sake of comparison, in Fig. $4 \mathrm{~b}$ we show a single-shot record of the downconverted image that was obtained upon removal of diffuser $\mathrm{D}_{1}$. The upper half of the CCD sensor $\left(\mathrm{P}_{\mathrm{FT}}\right.$ plane) is also shown in Fig. $4 \mathrm{~b}$ and, in this region, the 8 bit gray scale covers the intensity values from zero to the most intense pixel which identifies the $\boldsymbol{k}_{1}$ wave-vector carrying maximum intensity in the laser beam. Note that all images from computed correlations carefully recover the single shot image in Fig. 4b, the lower half sensor. On going from Fig. 2a to Figs. 3 and $4 a$ the noise in the reconstructed double-slit images decreases whereas the mean background value remains constant, being $\sim 0.2$ over a rectangle of $60 \times$ 15 pixels in the midpoint of the blank between the slits. In this region the background standard deviation reduces from the value 0.02 in Fig. 2a to 0.01 in Fig. 3, without sensible further decrease in Fig. 4a. The shifting and averaging procedure that brings from the single spatial intensity correlation over 6,000 records, such as that mapped in Fig. 2a, to any of the $G_{i, 231}^{\mathrm{AVE}}, G_{i, 232}^{\mathrm{AVE}}$ and $G_{i, 233}^{\mathrm{AVE}}$ in Fig. 3, greatly reduces the noise of the correlated image: slit profiles with minimum noise are obviously those recovered by $G_{i}^{\mathrm{FINAL}}$ as it is evident in the 3D plot in Fig. 5a. This figure shows the same data as in Fig. 4a and should be compared to the 3D plot in Fig. 5b, which shows the same data as in Fig. 2a. However, we consider these improvements in the quality of the recovered image as minor

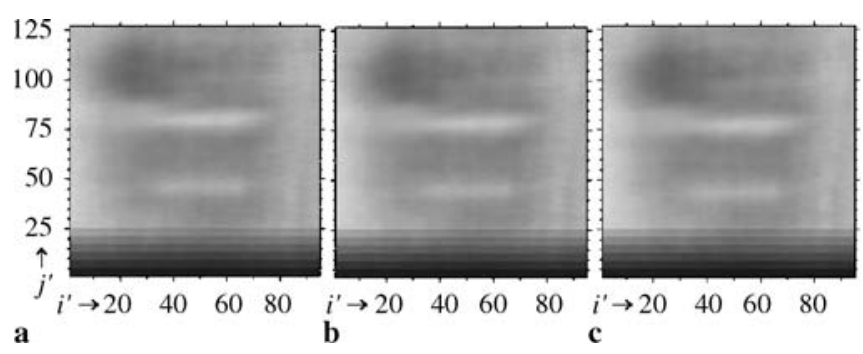

FIGURE 3 Averaged spatial correlation maps $G_{i, 231}^{\mathrm{AVE}}, G_{i, 232}^{\mathrm{AVE}}$, and $G_{i, 233}^{\mathrm{AVE}}$ in (a), (b), and (c), respectively displayed on an 8 bit gray scale covering the range from 0 to 0.346 

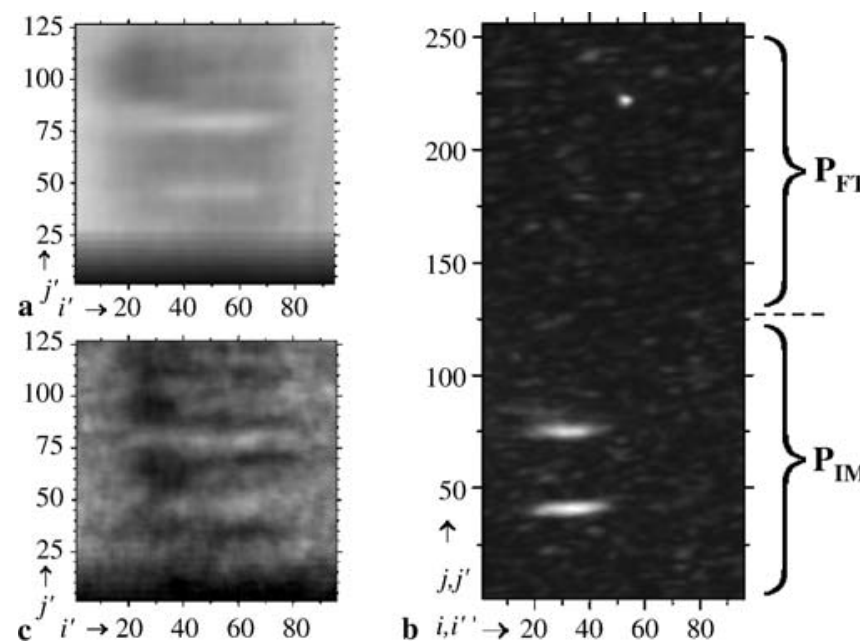

FIGURE 4 Map of $G_{i}^{\mathrm{FINAL}}$ in (a) as obtained by averaging $G_{i, 232}^{\mathrm{AVE}}, G_{i, 231}^{\mathrm{AVE}}$ and $G_{i, 233}^{\mathrm{AVE}}$ upon shifting the latter ones by \pm 1 ; (b) displays the intensity map of a single-shot downconverted image recorded without a diffusing plate on

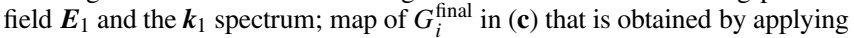
the same procedure as in (a) on $G_{i, 232}^{\text {ave }}, G_{i, 231}^{\text {ave }}$ and $G_{i 233}^{\text {ave }}$, which are calculated by using spatial intensity correlations of ensembles of 250 recorded intensity maps instead of 6000 , as for $G_{i, 232}^{\mathrm{AVE}}, G_{i, 231}^{\mathrm{AVE}}$ and $G_{i, 233}^{\mathrm{AVE}}$. The gray scales in (a) and (c) are as in Fig. 3. In (b), the gray scales are independently normalized to the maximum values in the upper $\left(\mathrm{P}_{\mathrm{FT}}\right)$ and lower $\left(\mathrm{P}_{\mathrm{IM}}\right)$ parts

compared to the value of the two steps above, involving shifts and averages, as a resource to recover images when spatial intensity correlations with the Fourier component intensity recorded in any of the $\mathrm{P}_{\mathrm{FT}}$ pixels fail. This is the case of spatial intensity correlations calculated on ensembles containing 250 records, which are too noisy to allow detection of the images (see $G_{i, 231}$ in Fig. 2b). To evaluate such a potential we threw 250 records out of the previous 6000 and used this reduced ensemble to calculate the spatial correlations according to (1) for the same $i$ - and $j$-values/ranges as above. We repeated the shifts-and-averages procedure of our extended protocol and obtained the $G_{i}^{\text {final }}$ map that is shown in Fig. 4c. Note that the double slit image is clearly detectable in Fig. 4c and fits the single-shot image of Fig. $4 \mathrm{~b}$. The $G_{i}^{\text {final }}$ data are also displayed in the $3 \mathrm{D}$ plot in Fig. $5 \mathrm{c}$ to show that the overall quality of the image recovered by $G_{i}^{\text {final }}$ is almost as good as that recovered by the intensity correlation over 6000 samples which is plotted in Fig. 5a. This is not surprising as the datum in a single pixel $\left(i^{\prime}, j^{\prime}\right)$ of Fig. 2a contains data from 6000 measurements in the same pixel and a single pixel of $G_{i}^{\text {final }}$ contains the information of 21 spatial intensity correlation maps calculated in parallel by averaging over 250 records, that is the information originally acquired in $250 \times 21=5250$ pixels of the recorded maps, namely $250 \times 21$ data in $\mathrm{P}_{\mathrm{IM}}$. In fact the datum in pixel $\left(i^{\prime}, j^{\prime}\right)$ of $G_{i}^{\text {final }}\left(i^{\prime}, j^{\prime}\right)$ is constructed by using 250 acquisitions in pixel $\left(i^{\prime}, j^{\prime}+\delta^{\prime}\right)$ with seven values of $\delta^{\prime}$, being $\delta^{\prime}=(4 / 3) \delta$ and $\delta=0,3,6, \ldots, 18$, for each $j^{\prime}-1, j^{\prime}, j^{\prime}+1$.

\section{4}

\section{Conclusion}

When the $\langle\ldots\rangle$ operation in (1) is carried out on the ensemble of 6000 frames, decoding the random frequencydownconverted images is accomplished by the simple calculation of $G_{i, j}$. When the ensemble reduces to 250 frames,
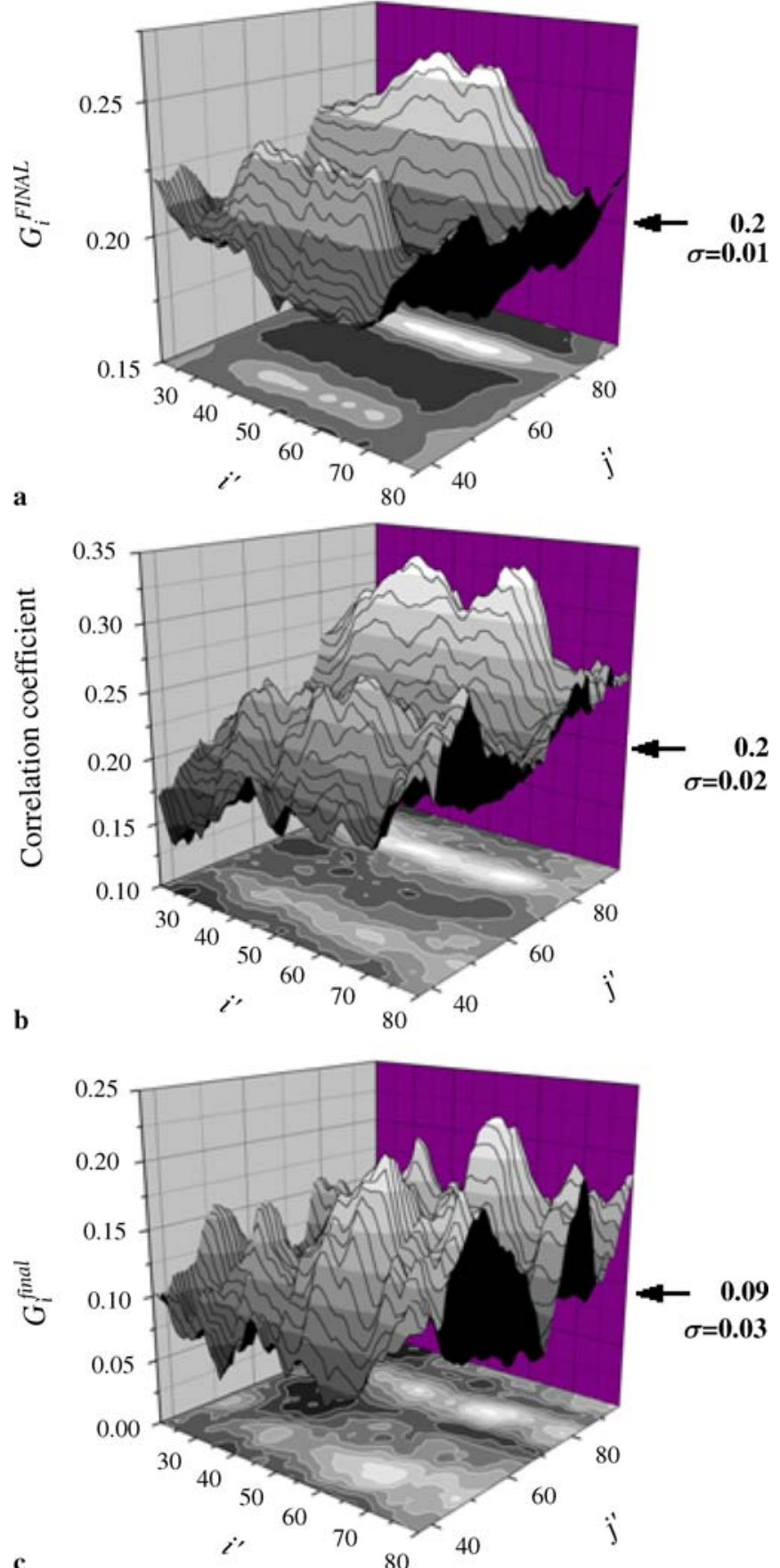

FIGURE 5 Maps of: (a) $G_{i}^{\mathrm{FINAL}}$, same data as in Fig. 4a; (b) spatial intensity correlation coefficients, $G_{i, 231}$, same data as in Fig. $2 \mathrm{a}$; $(\mathbf{c}) G_{i}^{\text {final }}$, same data as in Fig. 4c. The arrows mark the mean background values ( $\sigma$, standard deviation) in the rectangle $i^{\prime}=20$ to $79, j^{\prime}=54$ to 68 for $\mathbf{a}$ and $\mathbf{b}$ and $j^{\prime}=55$ to 69 for $\mathbf{c}$

such a task is only achieved upon calculating $G_{i}^{\text {final }}$. These further steps, which can be applied to both type I and type II frequency-downconversion processes, involve shifts that can be correctly performed only if $d$ and $f_{2}$ are known. If instead of the shot-to-shot recording of the Fourier transform of field $\boldsymbol{E}_{1}$ as it is when it leaves the BBO crystal after a weak interaction, field $\boldsymbol{E}_{1}$ first encounters an optical system and then meets the FT lens $\mathrm{L}_{2}$, more parame- 

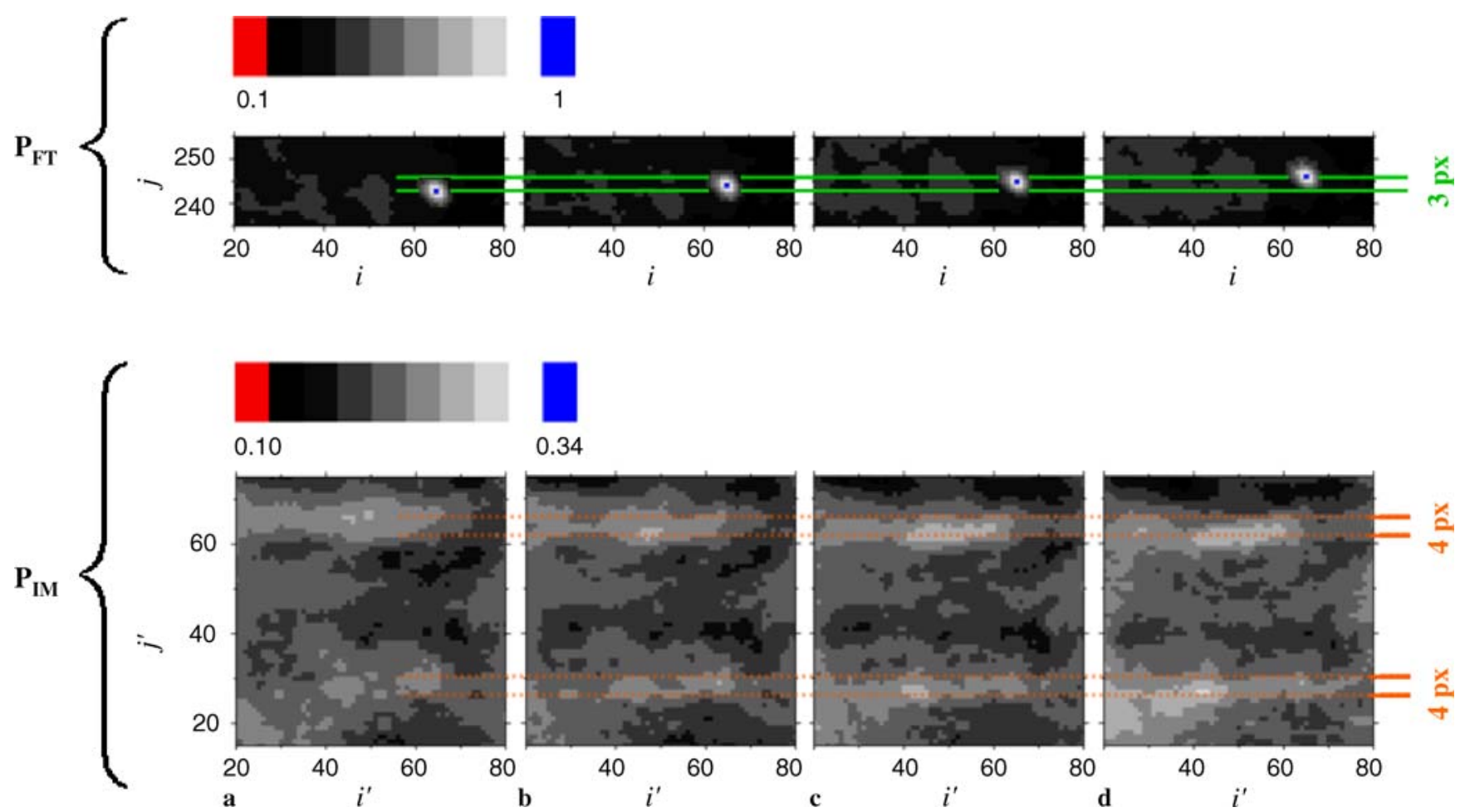

FIGURE 6 Maps of spatial intensity correlation coefficients. From (a) to (d): enlarged views of $G_{i, 242}, G_{i, 243}, G_{i, 244}$ and $G_{i, 245}$ (with $i=64$ ) obtained by applying (1) to an ensemble of 6000 single-shot CCD records. Upper panels, $\mathrm{P}_{\mathrm{FT}}$ : value 1 is only achieved by the autocorrelated pixel, which shifts from position $(64,242)$ in (a) to $(64,245)$ in $(\mathbf{d})$. Lower panels, $\mathrm{P}_{\mathrm{IM}}$ : reconstructed images with superimposed red lines at 4-pixel distance. The images shift in the down direction on going from a to $\mathbf{d}$ in agreement with (2)

ters, beyond $d$ and $f_{2}$, should be known to implement the protocol.

In conclusion, we think that the protocol presented here can be used as it is to reconstruct the far-field pattern of the object if one records the Fourier transform of field $\boldsymbol{E}_{2}$ instead of the intensity map of the real (chaotic) image. The entire procedure should work also for ghost imaging in the "macroscopic realm" of intensities [11]. In particular, we think that seeded frequency-downconversion can develop into a secure technique for sharing information having the parallel format typical of images/pictures. Work is in progress at our laboratory to demonstrate a secure transmission protocol based on the image recovery technique described in this work.

\section{Appendix}

Figure 6 displays the maps of $G_{i, j}$ (with $i=64$ ) for $j=242$, $243,244,245$ in panels a to $d$. They are enlarged to pixel resolution: while $\Delta j$ changes by 3 (see spacing of three pixels of the two green lines), the two reconstructed slits shift by four pixels (spacing of the red lines). Note that the slit spacing is 36 pixels.

ACKNOWLEDGEMENTS The authors are grateful to Fabio Paleari for kindly manufacturing the double slit. This work was partially sup- ported by I.N.F.M. through Project "PRA CLON" and by the Italian Ministry for University Research through the FIRB Project n. RBAU014CLC 002 .

\section{REFERENCES}

1 R.S. Bennink, S.J. Bentley, R.W. Boyd, Phys. Rev. Lett. 89, 113601 (2002)

2 D. Zhang, Y.-H. Zhai, L.-A. Wu, X.-H. Chen, Opt. Lett. 30, 2354 (2005)

3 M. D’Angelo, A. Valencia, M.H. Rubin, Y. Shih, Phys. Rev. A 72, 013810 (2005)

4 E. Puddu, A. Allevi, A. Andreoni, M. Bondani, Opt. Lett. 30, 1294 (2005)

5 F.T. Arecchi, Phys. Rev. Lett. 15, 912 (1965)

6 M. Bondani, E. Puddu, A. Andreoni, J. Mod. Opt. 53, 761 (2006)

7 M. Bache, E. Brambilla, A. Gatti, L.A. Lugiato, Phys. Rev. A 70, 023823 (2004)

8 M. Bache, E. Brambilla, A. Gatti, L.A. Lugiato, Opt. Express 12, 6067 (2004)

9 M. Bondani, A. Andreoni, Phys. Rev. A 66, 033805 (2002)

10 The movie displays the sequence of 21 spatial intensity correlation maps, from $G_{i, 231}$ to $G_{i, 251}$ (abscissas: $i=1$ to 96 ). In the upper part of each frame ( $j=128$ to 255 , region of $\left.P_{\mathrm{FT}}\right)$ the 8 bit gray scale covers the correlation values from 0.55 to 1 . Value 1 is only achieved by the autocorrelated pixel. In the lower part of each frame $(j=0$ to 127 , region of $\left.P_{\mathrm{IM}}\right)$ the 8 bit gray scale covers the correlation values from 0 to 0.45 . The single marker, in green, tracks the auto-correlated pixel and moves from $j=231$ to 251 at a speed which is $3 / 4$ the speed at which the couple of red markers moves. Note that the red markers remain synchronous with the slit images. Enlarged views of $G_{i, 242}, G_{i, 243}, G_{i, 244}$ and $G_{i, 245}$ (with $i=64)$ are displayed in Fig. 6 in the Appendix

11 A. Gatti, E. Brambilla, L.A. Lugiato, Phys. Rev. Lett. 90, 133603 (2003) 